\title{
Progressive Changes in the Microstructure and Texture in Pearlitic Steel during Wire Drawing
}

\author{
Pankaj KUMAR, Nilesh Prakash GURAO, (1) Arunansu HALDAR ${ }^{21}$ and Satyam SUWAS ${ }^{1)}$ \\ 1) Department of Materials Engineering, Indian Institute of Science, Bangalore, 560012 India. E-mail: satyamsuwas@materials. \\ iisc.ernet.in 2) TATA Steel R\&D, Jamsedpur, India.
}

(Received on November 30, 2010; accepted on January 13, 2011)

\begin{abstract}
The evolution of microstructure and texture during wire drawing of pearlitic steel was studied using scanning electron microscopy and X-ray diffraction. Wire drawing induces elongation of ferrite grains with the development of $\langle 110\rangle$ fibre texture. The cementite lamellae also have a tendency to reorient themselves along the wire axis with increasing strain. Viscoplastic self-consistent simulations were employed to simulate the texture evolution in the ferrite phase.
\end{abstract}

KEY WORDS: pearlitic steel; wire-drawing; viscoplastic self consistent simulations.

\section{Introduction}

Cold drawn pearlitic wire has a great practical importance in structural applications, like suspension cables, tire cord and springs, where high strength is required, together with acceptable level of toughness. It has been reported that during wire drawing, the mechanical properties of the steel substantially improve. ${ }^{1-10)}$ The improvement of properties can be correlated with the changes occurring during the drawing operation of pearlitic steel. This has been attributed to the structural refinement occurring during the wire drawing operation. During wire drawing, the interlamellar thickness decreases proportionally which further cause increase in the strength of the steel. ${ }^{6-10)}$ In addition, the presence of cementite greatly influences the mechanical properties of pearlitic steel. Cementite is hard and brittle phase in pearlitic steel that contributes to strengthening. The harder cementite phase co-deforms with the softer ferrite during wire drawing. Several studies have been reported on the plastic deformation of cementite. ${ }^{7-13)}$ It appears that high hydrostatic pressure during the drawing process favors the plastic deformation of cementite. ${ }^{10)}$ However, there is no comprehensive report on the overall (gross) plastic deformation of pearlite. It is, however, reported that the pearlitic colony rotates and aligns along the wire axis. In addition, cementite lamellae were found to bend and stretch to align with the wire axis. During the drawing process, progressive evolution of texture occurs in the material. Ferrite phase develop $<110>$ type fibre texture during the drawing operation. ${ }^{11-13)}$ Despite the numerous studies carried out to understand the deformation behaviour of pearlitic steels during wire drawing, an investigation complementing experimental findings with crystal plasticity based simulations is still warranted to understand the micro-mechanisms of pearlite deformation. Viscoplastic self-consistent simulations based on Eshelbey's inclusion problem ${ }^{14,15)}$ provide a robust tool to study texture evolution in complex multi-phase materials like pearlitic steels.

In the present investigation, viscoplastic self-consistent simulations were carried out along with experimental studies on the progressive changes occurring in the microstructure and texture of pearlitic steel during wire drawing to develop a comprehensive understanding of the process. The evolution of microstructure was monitored using a scanning electron microscope and texture measurement was carried out using X-ray diffraction. Viscoplastic Self-consistent (VPSC) simulation were performed to simulate texture evolution and hence, to understand the micro-mechanism of deformation of ferrite phase during the wire drawing operation.

\section{Experimental Procedure}

The present study was carried out on pearlitic steel provided by TATA Steel Ltd., India. This material was commercially produced fully pearlitic steel, in the as-rolled condition. The detailed chemical composition of the steel is given in Table 1. The initial material was characterized by random texture of ferrite phase. The microstructure of the as received material had non-uniform grain size for pearlite as well as non-uniform cementite lamella thickness.

In order to explore the progressive microstructural and texture changes in pearlitic steel, samples from five intermediate stages of the entire wire drawing operation were considered. The initial perform for wire drawing was in the form of circular rod of diameter $7.56 \mathrm{~mm}$. This sample was

Table 1. Chemical composition of as received sample.

\begin{tabular}{cccccccc}
\hline Composition & $\mathrm{C}$ & $\mathrm{Mn}$ & $\mathrm{S}$ & $\mathrm{P}$ & $\mathrm{Al}$ & $\mathrm{Cu}$ & $\mathrm{Cr}$ \\
\hline $\mathrm{Wt} \%$ & 0.8 & 0.63 & 0.1 & 0.014 & 0.042 & 0.005 & 0.053
\end{tabular}


passed through several dies at room temperature under dry condition. During each stage the true strain is given by,

$$
\varepsilon=\ln \left(\mathrm{d}_{0} / \mathrm{d}\right)
$$

Where, $\mathbf{d}_{\mathbf{0}}$ and $\mathbf{d}$ are initial and final diameter respectively.

The parameters characterizing deformation at each stage are given Table 2. Microstructures of the wire drawn samples at successive strain levels were examined over the longitudinal wire section. The samples were cut, mounted and mechanically polished following standard metallographic practice. The microstructure was revealed using Acid Picral (4\% picric acid and $96 \%$ ethanol). Microstructures were recorded using a Field Emission Gun scanning electron microscope (FEG SEM) Sirion. Hardness tests were performed on the longitudinal wire section using Vickers hardness tester with a load of $1 \mathrm{~kg}$. Five readings were taken for each case and the mean value was obtained.

Table 2. Drawing reduction per step for pearlitic steel.

\begin{tabular}{ccccccc}
\hline Drawing Stage & 0 & 1 & 2 & 3 & 4 & 5 \\
\hline $\mathrm{d}(\mathrm{mm})$ & 7.56 & 6.67 & 6.04 & 5.4 & 4.89 & 4.04 \\
Strain impartd $(\varepsilon)$ & 0 & 0.12 & 0.22 & 0.33 & 0.43 & 0.63 \\
\hline
\end{tabular}

Bulk texture measurements were performed for the ferrite phase using X-ray diffraction with Co K $\alpha$ radiation on the same longitudinal section of the samples. Three incomplete pole figures namely $\{110\},\{200\}$, and $\{211\}$ were measured using Bruker D8: Discover texture goniometer based on Schultz reflection geometry. The data obtained from the pole figures was used to calculate orientation distribution function (ODF) following arbitrary defined cells (ADC) algorithm $^{16)}$ used in the commercially available software Labosoft. The inverse pole figures and volume fractions of the texture components were also calculated from the ODFs.

\section{Simulations}

A Visco Plastic Self-Consistent code VPSC $7 b^{14,15)}$ was used to simulate texture evolution in the ferrite phase during wire drawing operation. Single phase simulations were employed as the cementite phase shows brittle behaviour at room temperature.

The behavior of a single grain can be given by a rate dependent viscoplastic law as:

$$
D_{i j}^{c}=\dot{\gamma}^{0} \sum m_{i j}^{S}\left(\frac{m^{s}: \sigma}{\tau^{S}}\right)^{n}
$$

Equation (1) represents nonlinear function. This equation can be expressed in pseudo-linear form:

$$
D_{i j}^{c}=\dot{g}^{0} \sum m_{i j}^{S} m_{k l}^{S}\left(\frac{m^{s}: s}{t^{s}}\right)^{n} s_{k l}=M_{i j k l}^{c} s_{k l}^{c}
$$

where $D^{\mathrm{c}}$ is strain rate $\dot{\gamma}^{0}$ a normalizing strain rate, $\mathrm{s}$ is a label for the slip systems, $\mathrm{m}^{\mathrm{s}}$ is the geometric Schmid tensor, $\tau^{\mathrm{s}}$ is a threshold stress of a slip system and $\mathrm{n}$ is the inverse rate sensitivity. $M_{i j k l}^{c}$, the secant visco-plastic compliance tensor of the grain and depends on the stress state.
Slip will occur in a system $\mathrm{s}$ when the resolved shear stress $\mathrm{m}^{\mathrm{s}}: \sigma^{\mathrm{c}}$ approaches the critical value $\tau^{\mathrm{s}}$. Hence, $\tau^{\mathrm{s}}$ describe the resistance for activation of slip and it usually increases with deformation and it is given by the extended Voce law:

$$
\tau^{S}(\Gamma)=\tau_{0}+\left(\tau_{1}+\theta_{1} \Gamma\right)\left[1-\exp \left(\frac{-\theta_{0} \Gamma}{\tau_{1}}\right)\right]
$$

Where $\Gamma=\int_{0}^{t} \sum_{S}\left|\dot{\gamma}^{S}\right| d t$ is the accumulative shear in the grain; $\tau_{0}, \tau_{1}, \theta_{0}$ and $\theta_{1}$ contribute to the hardening parameter for the slip system.

From Eq. (2) at aggregate level the overall strain rate and stress can be related through a secant constitutive law as:

$$
\bar{D}=\overline{M^{\mathrm{sec}}}: \bar{\sigma}
$$

Solution of this equation for an inhomogeneous grain embedded in HEM leads to:

$$
\left(D^{c}-\bar{D}\right)=\tilde{M}^{c}:\left(\sigma^{c}-\bar{\sigma}\right)
$$

Where $\tilde{M}^{c}$ is the interaction tensor given by

$$
\tilde{M}^{c}=n^{\text {eff }}(I-E)^{-1}: E: \bar{M}^{c} ; E \text { is the Eshelby tensor. }
$$

The algorithm enables one to choose the slip systems, rate sensitivity, hardening parameter etc. Once the activity of slip systems is obtained, grain rotation could take place that leads to the texture evolution. A rate sensitivity of 0.05 was used for the activated slip system.

The texture of the ferrite phase measured for the initial sample was discretized using the Labotex software in 2000 single orientations. These discretized orientations were used as input to the code. It was assumed that at room temperature $\{110\}<1 \overline{1} 1>$ and $\{112\}<\overline{1} 1 \overline{1}>$ slip systems are the active slip systems. Table 3 shows the Voce parameters for the two slip systems used for the simulations.

Table 3. Voce hardening parameter used for the simulation.

\begin{tabular}{ccccc}
\hline Slip System & $\tau_{0}$ & $\tau_{1}$ & $\theta_{0}$ & $\theta_{1}$ \\
\hline$\{110\}<1 \overline{1} 1>$ & 10 & 30 & 5 & 3 \\
$\{112\}<\overline{1} 1 \overline{1}>$ & 15 & 45 & 3 & 1 \\
\hline
\end{tabular}

\section{Results}

\subsection{Evolution of Microstructure}

Figures 1 $(a-f)$ show the microstructure of the samples at different stages of wire drawing. The microstructure of starting material clearly shows randomly oriented pearlitic colonies of different sizes. The orientations of cementite lamellae in individual pearlitic colonies are also different. During wire drawing, the lamellae have a tendency to orient along the drawing axis. Bending and kinking of cementite lamellae was observed during the deformation of the material. This was accompanied with the elongation of pearlite colonies in the drawing direction. At higher strain, the extent of bending of cementite was found to increase. A closer inspection of the microstructures reveal that the cementite lamellae which were oriented parallel to the wire axis get thinned further leading to somewhat fibrous structure, while 

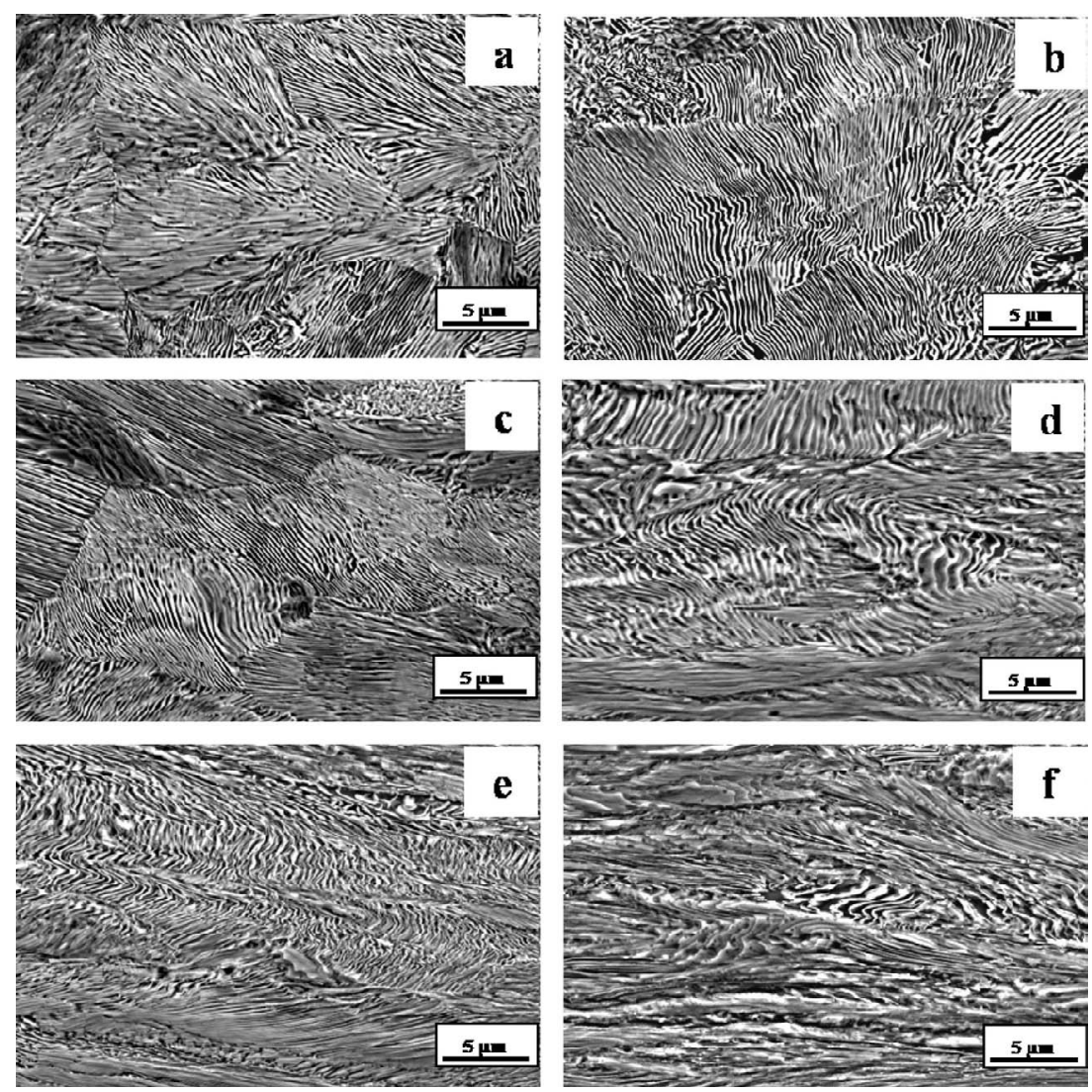

Fig. 1. High resolution scanning electron micrographs for the wire samples drawn to different stain levels (a) $\varepsilon=0$, (b) $\varepsilon=0.12$,(c) $\varepsilon=0.22$, (d) $\varepsilon=0.33$, (e) $\varepsilon=0.43$, (f) $\varepsilon=0.63$. The horizontal direction represents the wire drawing direction.

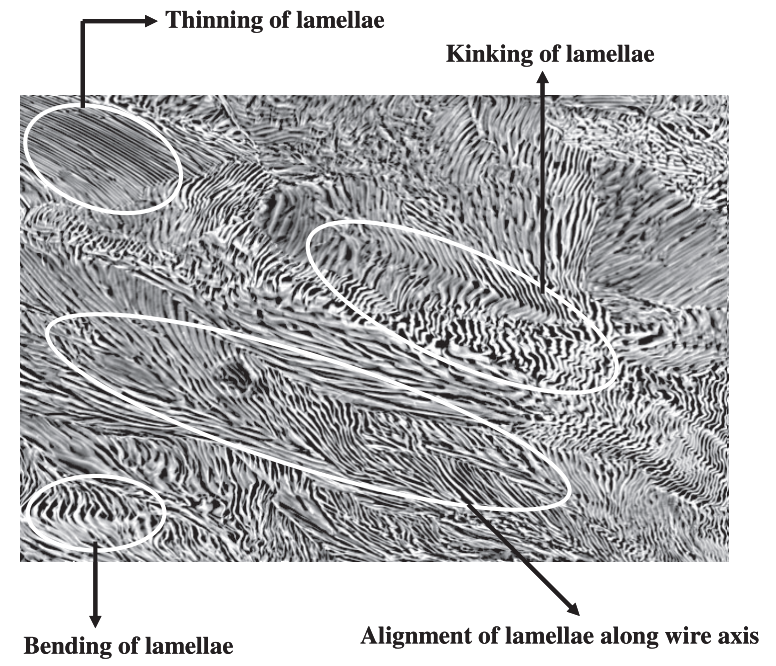

Fig. 2. Microstructural features representing different types of morphological changes during the wire drawing.

those oriented unfavorably get bent or kinked (Fig. 2). The variation of hardness with the variation of strain is plotted in Fig. 3. It can be seen that the hardness of the drawn wire increases monotonically with the strain.

\subsection{Evolution of Texture}

Texture measurement was carried out for the ferrite phase, where textural changes are more likely to occur due to the deformation. Texture of the starting material as well as the drawn wire at different strain levels has been present-

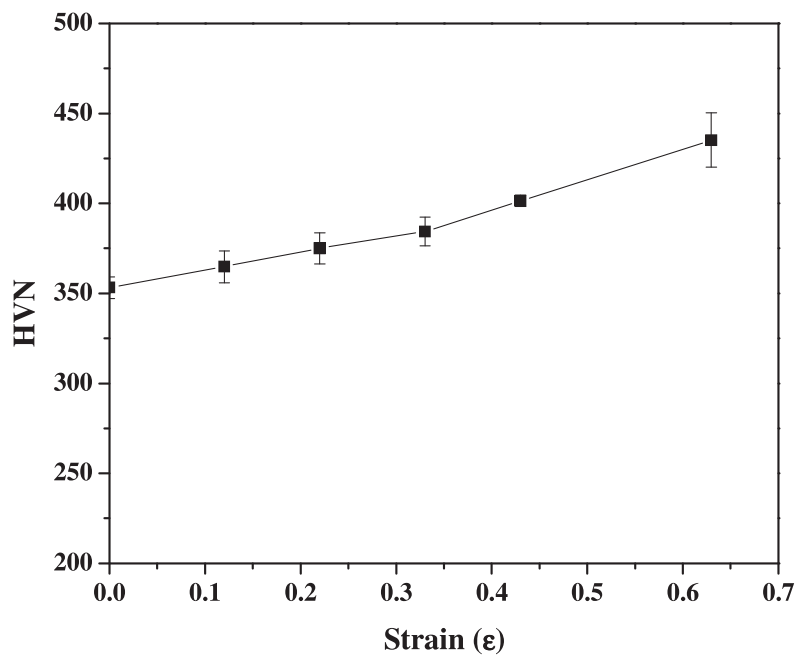

Fig. 3. Progressive change in the hardness of the samples during wire drawing.

ed in terms of inverse pole figure. Figure 4 shows the inverse pole figure along the wire drawing axis for the starting material as well as the drawn wires. It can be seen that starting material had an almost random texture. As the deformation proceeds, the wire axis starts getting clustered near the orientation $<110>$ of the stereographic triangle. The volume fraction calculations (Fig. 5) show that as the strain increases the volume fraction of $<110>$ fibre component increases. The volume fraction of $<110>$ fibre component, however, saturates after the $4^{\text {th }}$ pass. 

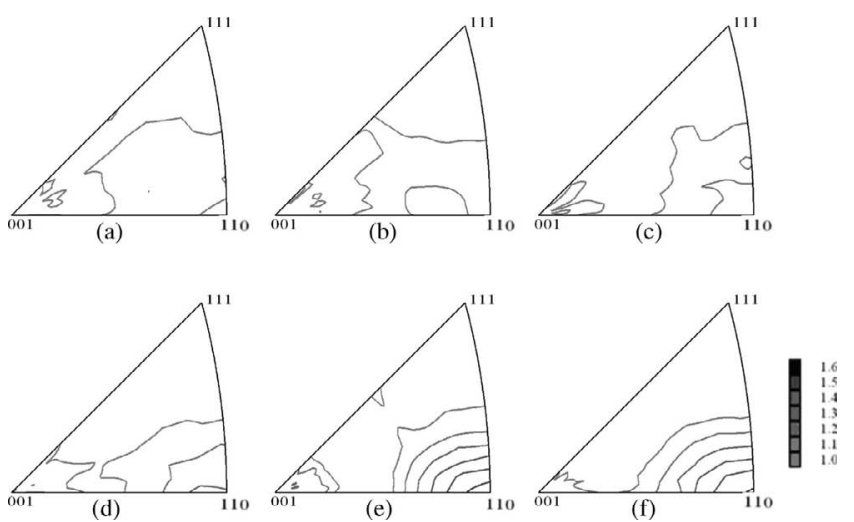

Fig. 4. Calculated 001 inverse pole figures for ferrite phase at (a) $\varepsilon=0$, (b) $\varepsilon=0.12$, (c) $\varepsilon=0.22$, (d) $\varepsilon=0.33$, (e) $\varepsilon=0.43$, (f) $\varepsilon=0.63$.

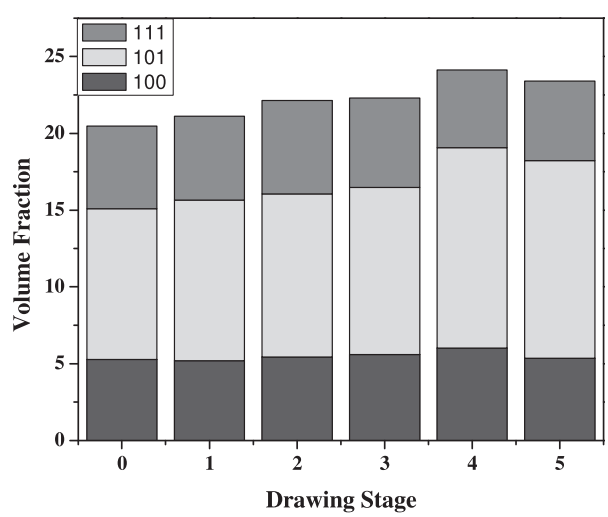

Fig. 5. Calculated volume fraction of important fiber texture components for ferrite phase during drawing.

The simulated textures (Fig. 6) show a reasonably good match with the experimental textures qualitatively. The difference in the experimental and simulated textures appears in terms of intensity.

\section{Discussion}

\subsection{Evolution of Microstructure}

The microstructure of initial material shows random distribution of pearlite colonies. In these colonies, the lamellae orientations are varying with respect to the wire axis. Some lamellae are orientated in the direction of wire drawing axis while the others differently. The microstructural development in the wire samples indicate that pearlitic colonies progressively align with the wire axis with increasing drawing strain. This is accompanied by the combination of bending and stretching of pearlite colony. The cementite lamellae, that are oriented in the direction of wire axis, stretch themselves and cause thinning while the unfavorably orientated lamellae try to reorient in the wire drawing direction. The schematic diagram of lamellae deformation has been shown in Fig. 7. The changes occurring in the morphology of the cementite lamellae like bending, kinking, thinning of the lamellae are consistent with the previous report. ${ }^{8-10,13)}$

It has been reported in the literature ${ }^{11)}$ that only two close packed directions $<111>$ and $<11 \overline{1}>$ contribute to the stretching of the wire out of four. During the drawing oper-

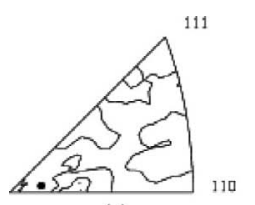

(a)
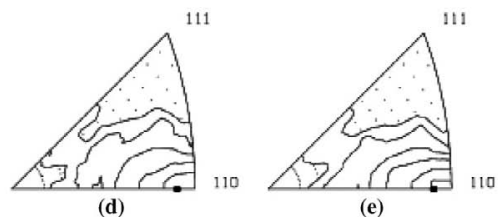

11

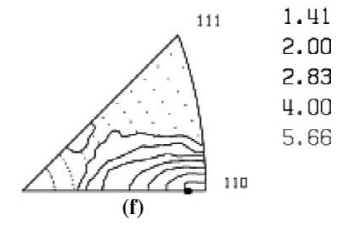

Fig. 6. Simulated 001 inverse pole figures for ferrite phase of the wires drawn to the strain levels (a) $\varepsilon=0$, (b) $\varepsilon=0.12$, (c) $\mathcal{E}=0.22$, (d) $\varepsilon=0.33$, (e) $\varepsilon=0.43$, (f) $\varepsilon=0.63$.

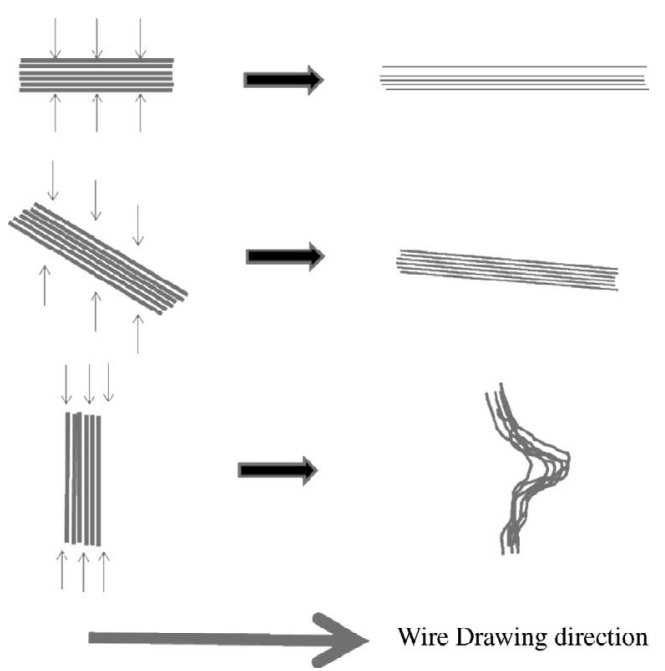

Fig. 7. Schematic diagram of cementite deformation during wire drawing.

ation, surface area of cementite lamellae increases. This might lead to the generation of misfit dislocations and accumulation of dislocations at the interface. As a result the stress level at the interface becomes quite high. This can cause the deformation of cementite lamellae. Van Acker et $a l{ }^{12)}$ reported that the interlamellar stress at the interface can reach as high as $2000 \mathrm{MPa}$. The activation of additional $\{110\}<111>$ in ferrite and $\{100\}$ slip in cementite causes accumulation of misfit dislocation at the interface. ${ }^{13)}$ The generation of interface dislocation during deformation can also be explained on the basis of strain gradient plasticity theory. ${ }^{17)}$ It has also been observed that, lamellae parallel to the wire axis get deformed and fragment at higher strain. This is similar to the deformation of tube under uni-axial tension. It is well studied and reported that during wire drawing, the thickness of lamellae decreases. With decrease in lamellae thickness, elastic stresses from the two opposing inter-phase boundaries overlap resulting elastic stresses at the interface. ${ }^{13)}$ This stress cause the deformation of axially oriented lamellae.

The above mentioned microstructural features have a close relationship with the variation of mechanical properties. Earlier investigations have shown that the yield stress increases with the decrease in the interlamellar spacing dur- 
ing wire drawing. ${ }^{6-9)}$ Therefore, the phenomena of increase in the hardness with the strain may be attributed to the alignment of cementite lamellae into the wire direction, consequently decreasing the interlamellar spacing during wire drawing of the steel.

\subsection{Texture Evolution}

The results of the investigation suggest that the wire drawing operation leads to the formation of $<110>$-type fibre texture in ferrite phase. Many investigations have reported that the ferrite grains tend to rotate towards the wire axis along with the cementite lamallae and form axial $<110>$ fibre. It is observed that the $<110>$ texture component is saturates at higher strain. The phenomena of saturation of texture component can be attributed to the presence of cementite. The presence of non-deforming hard phase affects the rotation of ferrite during the deformation. The imposed deformations were accommodated in ferrite phase by activation of $\{110\}<1 \overline{1} 1>$ and $\{112\}<\overline{1} 1 \overline{1}>$ slip systems. The activity of the slip systems involved during the drawing process as obtained from simulation is shown in Fig. 8. It depicts that during the $\{110\}<1 \overline{1} 1>$ slip systems dominates during the course of deformation.

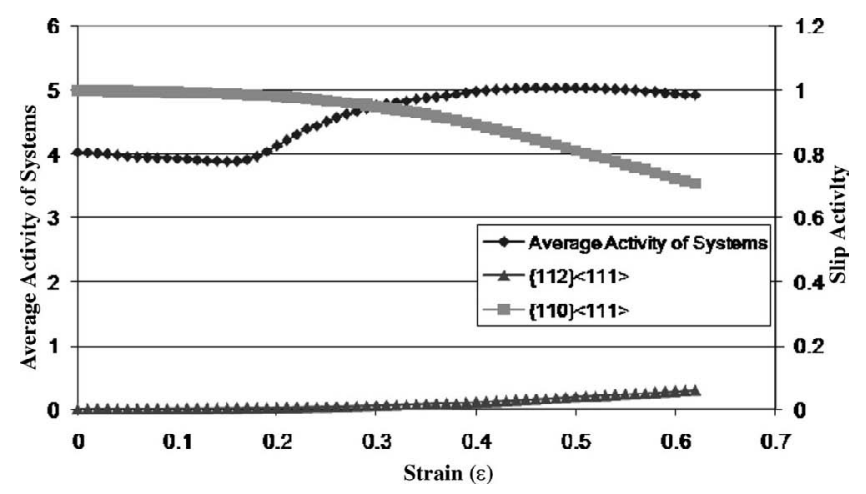

Fig. 8. Simulated activity of the slip systems of ferrite phase during the wire drawing.

During initial stages of deformation, $\{110\}<1 \overline{1} 1>$ slip system is active. The activity of this system remains constant till a strain $\varepsilon \sim 0.2$ after which it decreases. The number of average active slip systems shows a gradual decrease with deformation till the same value of strain $\varepsilon \sim 0.2$. At higher strain $(\varepsilon>0.2),\{112\}<\overline{1} 1 \overline{1}>$ slip system gets activated. This leads to an increase in the number of average active slip systems till a strain of $\varepsilon \sim 0.4$. With further increase in strain, the number of average active slip systems remains almost constant though there is an increase in the activity of the $\{112\}<\overline{1} 1 \overline{1}>$ slip system at the expense of the $\{110\}<1 \overline{1} 1>$ slip system. At higher strain $(\varepsilon \sim 0.6)$, there is a slight decrease in the number of average active slip systems that can be attributed to evolution of strong geometrical anisotropy in the microstructure due to elongated grains. It has been reported earlier that the Relaxed constraint model is applicable in materials at higher strains instead of the Full Constraint Taylor model that is relevant during initial stages of deformation. The elongation of grains relaxes the criterion of 5 independent slip systems to accommodate plastic deformation in cubic materials and the imposed deformation can be incorporated by activation of less than five independent slip systems. ${ }^{18,19)}$ The viscoplastic self-consistent model that can be tuned to get a micro-mechanical response between the two extremes models is able to capture this effect as the shape of the ellipsoids is updated after every step in simulations.

It is possible that the hard non-deforming cementite restrict the rotation of ferrite locally causes weakening texture intensity. Such a weakening of the texture of softer phase in two phase materials has been reported earlier. ${ }^{20,21)}$ The simulation result also shows the formation of $<110>$ fibre texture, however, the intensity level is higher compared to the experimental texture.

\section{Conclusions}

Pearlitic steel was subjected to wire drawing to a strain $\mathcal{E} \sim 0.63$ and the resulting microstructure and texture were determined. Based on the experimental results following conclusion can be drawn:

Wire drawing of pearlitic steel is characterized by significant evolution of microstructure for the ferrite and cementite phase individually as well as for the pearlitic colonies. The pearlitic colonies whose cementite lamellae are oriented perpendicular to the wire axis exhibit kinking and bending of the lamellae, while the colonies having lamellae parallel to the wire axis gets thin and form fibrous structure. The ferrite phase shows an evolution of $\langle 110\rangle$ fibre texture with increase in strain. Viscoplastic self-consistent simulations are able to successfully capture the deformation texture evolution in the ferrite phase during wire drawing.

\section{Acknowledgements}

The authors duly acknowledge TATA Steel Ltd., India for providing the financial grant and the material to carry out the experiments. Thanks are due to Dr. D. Bhattacharjee for his constant encouragement at various level stages of this project. The microstructural characterization was carried at the Institute Nano-Science Initiative and bulk texture measurement was carried at X-ray centre at the Indian Institute of Science, Bangalore. The authors acknowledge the support of Department of Science and Technology, Government of India, for providing these facilities through FIST (DSTFIST) program.

\section{REFERENCES}

1) H. G. Paris: Metallurgy, processing and application of Metal wires.

2) J. J. Lewandowski and A. W. Thompson: Metall. Trans., 17A (1986), 461.

3) J. J. Lewandowski and A. W. Thompson: Metall. Trans., 17A (1986), 1769.

4) D. J. Alexander and I. M. Bernstein: Metall. Trans., 20A (1989), 2321.

5) A. Inoue, T. Ogura and T. Masumoto: Scr. Metall., 11 (1976), 1.

6) D. A. Porter, K. E. Eastarling. and G. D. W. Smith: Acta Metall., 26 (1978), 1405.

7) J. J. Pepe: Metall. Trans., 4A (1973), 2455.

8) G. Langford: Metall. Trans., 8A (1977), 861.

9) W. J. Nam, C. M. Bae, S. J. Oh and S. J. Kwon: Scr. Mater., 42 (2000), 457.

10) G. Langford: Metall. Trans., 1B (1970), 465

11) W. F. Hosford Jr.: Trans. ASM-AIME, 230 (1964), 12.

12) K. Van Acker, J. Root, P. Van Houtte and E. Acrnoudt: Acta Mater., 44 (1996), 4039.

13) Michael Zelin: Acta Mater., 50 (2002), 4431. 
ISIJ International, Vol. 51 (2011), No. 4

14) R. A. Lebensohn and C. N. Tome: Acta Metall. Mater., 41(1993), 2611

15) C. N. Tome, G. R. Cannova and U. F. Kocks: Acta Metall., 32 (1984), 1637.

16) K. Pawlik: Phys. Stat. Sol. B, 134 (1986), 477.

17) R. K. Abu Al-Rub and G. Z. Voyiadjis: Int. J. plasticity, 22 (2006), 654.
18) H. Honneff and H. Mecking: Proc. 6th Int. Conf. on Texture of Materials, (1981) 347.

19) P. Van Houtte: Mater. Sci. Eng., 55 (1982) 69.

20) S. Suwas, A. K. Singh, R. K. Ray and S. Bhargava: Scr. Mater., 35 (1996), 897.

21) M. Kiran Kumar, I. Samajdar, N. Venkatramani, G. K. Dey, R. Tewari, D. Srivastava and S. Banerjee: Acta Mater., 51 (2003), 625. 